\title{
Multi-focal optical-resolution photoacoustic microscopy in vivo
}

\author{
Liang Song, Konstantin Maslov, and Lihong V. Wang* \\ Optical Imaging Laboratory, Department of Biomedical Engineering, Washington University in St. \\ Louis, St. Louis, Missouri 63130
}

\section{Abstract}

Although ultrasound arrays were exploited in photoacoustic imaging to improve imaging speed, ultrasound-array-based optical-resolution photoacoustic microscopy (OR-PAM) has never been achieved previously. Here we present our development of multi-focal OR-PAM using a microlens array for optical illumination and an ultrasound array for photoacoustic detection. Our system is capable of imaging hemoglobin concentration and oxygenation in individual microvessels in vivo at high speed. Compared with a single focus, multiple foci reduce the scanning load and increase the imaging speed significantly. The current multi-focal system can acquire $1000 \times 500 \times 200$ voxels at $\sim 10-\mu \mathrm{m}$ lateral resolution within $4 \mathrm{~min}$.

Due to the vital role of the microcirculation in the regulation of hemodynamics and metabolism, imaging of the microvasculature is crucial to both clinical practice and preclinical research [1]. Many high-resolution optical imaging modalities-including confocal microscopy, two-photon microscopy, and optical coherence tomography-have been adopted for microvascular imaging [2-4]. However, they either require exogenous fluorescent agents or have insufficient sensitivity to image a single capillary. In addition, none of them can accurately assess the oxygen saturation $\left(\mathrm{sO}_{2}\right)$ of hemoglobin-an important functional parameter in disease diagnosis.

In contrast, optical-resolution photoacoustic microscopy (OR-PAM) provides extremely high sensitivity to optical absorption, with optical diffraction limited lateral resolution [5]. It is capable of imaging both the total concentration and $\mathrm{sO}_{2}$ of hemoglobin in microvesselsincluding capillaries - in vivo, making it a viable tool for microvascular imaging [6]. However, the imaging speed of OR-PAM using single-element ultrasonic detection is usually limited by mechanical scanning.

Previously, to improve the imaging speed of OR-PAM, a Galvo mirror was used to rapidly scan the illumination laser beam confined within the field of view of an unfocused ultrasonic transducer [7]. A hybrid optical-mechanical scanning OR-PAM using a cylindrically focused ultrasonic transducer was also developed [8]. However, since the ultrasonic transducer was either unfocused or focused in only one axis, the signal-to-noise (SNR) ratio of the system was inherently limited. In addition, because only a single laser beam and a single ultrasonic transducer were used, these systems demand a laser pulse repetition rate as high as $5-10 \mathrm{kHz}$ to significantly improve the imaging speed. Presently, lasers with such high pulse repetition rates usually lack the wavelength tunability needed for measuring $\mathrm{sO}_{2}$. Furthermore, although ultrasound arrays were used to improve the speed of photoacoustic imaging systems [9-10], and although optical focusing was used to improve the elevational resolution of such systems [11], none of them achieved optical resolution in both the lateral and elevational directions.

\footnotetext{
*Corresponding author: 1hwang@biomed.wustl.edu.
} 
In this work, we developed multi-focal optical illumination in conjunction with ultrasonic array detection to achieve both optical resolution and high speed for photoacoustic imaging. Figure 1 illustrates the principles of the multi-focal optical-resolution photoacoustic microscopy (MFOR-PAM). For photoacoustic excitation, a tunable dye laser (Cobra, Sirah, Germany), pumped by a Q-switched Nd:YLF laser (INNOSLAB, Edgewave, Germany), was used. The laser system (with Rhodamine 6G laser dye) had a peak output at $570 \mathrm{~nm}$ with a 20-nm tuning range, and was operated at a pulse repetition rate up to $1.3 \mathrm{kHz}$. To provide multi-focal optical illumination, a microlens array (11-1640-123-000, SUSS MicroOptics, Switzerland) was used. The microlens array consists of a $20 \times 40$ quadratic grid of spherical microlenses with $250-\mu \mathrm{m}$ center-to-center spacing. Each microlens has a diameter a few micrometers smaller than $250 \mu \mathrm{m}$ and an effective focal length $\sim 1.2 \mathrm{~mm}-$ which is also its working distance. In theory, it can produce a diffraction-limited focus with a radius of $\sim 4 \mu \mathrm{m}$ at waist and a Rayleigh length of $\sim 80 \mu \mathrm{m}$. In this study, a line of 20 microlenses from the microlens array was used, providing 20 focused optical illumination spots along $x$ (Figure 1). The distance between two nearby spots, determined by the pitch of the microlens array, was $250 \mu \mathrm{m}$, greater than the $\sim 80-\mu \mathrm{m}$ acoustic lateral resolution of our ultrasound array.

For ultrasonic detection, a linear ultrasound array was used, simultaneously detecting photoacoustic emissions from all illumination beams. The ultrasound array, with a center frequency of $30 \mathrm{MHz}$ and a receiving-only bandwidth of $70 \%$, consists of 48 elements (each has a dimension of $82 \mu \mathrm{m} \times 2 \mathrm{~mm}$ ) spaced $100 \mu \mathrm{m}$ along $x$. Further technical details about this array can be found in our previous publications $[10,12]$. To acquire a volumetric image, the sample was scanned in the $x-y$ plane. At each scanning position, the signals acquired from different illumination sites were reconstructed by using a photoacoustic delay-and-sum algorithm [10] followed by integrating the delay-and-summed signals into corresponding illumination lines. Of note, the use of the ultrasound array with photoacoustic reconstruction allows the spacing between adjacent optical illumination beams to be as small as the acoustic lateral resolution of the ultrasound array. Fundamentally different from simply combining multiple assemblies of a single optical focusing element and a single ultrasonic transducer, this approach enables us to position optical foci much closer to each other and potentially to scan optically at high speed.

Due to the simultaneous photoacoustic excitation from the 20 illumination beams, the number of scanning steps along $x$ was reduced 20-fold compared with that in conventional single-focus OR-PAM. In theory, this reduction could result in a 20 -fold increase in imaging speed. In our system, as a proof of concept, data from the 48-channel ultrasound array are multiplexed to an 8-channel data acquisition card (Octopus 8389, GaGe Applied Technologies, USA) [10, 12], reducing the possible speed increase by 6 times. However, even with the multiplexing, our MFOR-PAM system can acquire a data set of $1000 \times 500 \times$ 200 voxels within 4 min, three to four times faster than existing mechanical scanning singlefocus OR-PAM.

As measured previously, the axial resolution of our system, determined by the receiving ultrasonic bandwidth, is $\sim 25 \mu \mathrm{m}$ [12]. To quantify the lateral resolution, which is determined by the optical focus of the microlens array, we imaged two 6- $\mu \mathrm{m}$ diameter crossed carbon fibers, using a scanning step of $2.5 \mu \mathrm{m}$. Note that, after the implementation of optical focusing, the aforementioned lateral and elevational resolutions with respect to the ultrasound array have now become undistinguishable with respect to the imaging system. Hence, hereafter, both resolutions will be referred to as lateral resolution. Figure 2(a) shows a maximum amplitude projection (MAP) image of the two carbon fibers. In this report, all MAP images were formed by projecting the maximum photoacoustic amplitudes along the $z$ axis to the $x-y$ plane. Figure 2(b) shows the FWHM of the vertical fiber, demonstrating that 
the lateral resolution of our system is at least as fine as $10 \mu \mathrm{m}$. The contrast-to-noise ratio (CNR), measured from Fig. 2(a), is as high as $38 \mathrm{~dB}$.

To demonstrate the in vivo imaging capability of our system, we imaged the microvasculature in the right ear of an anesthetized nude mouse (Hsd, Athymic NudeFoxn1NU, Harlan Co.) weighing $\sim 20 \mathrm{~g}$. In order to couple the photoacoustic waves to the ultrasound array, a thin layer of ultrasonic gel was applied to the mouse ear. The image was acquired completely noninvasively, without using any optical clearing agent. After imaging, the animal naturally recovered without observable laser damage. All experimental animal procedures were carried out in compliance with protocols approved by the Animal Studies Committee of Washington University.

Figure 3(a) shows an in vivo MAP image of the mouse ear microvasculature acquired by our MFOR-PAM system at $570 \mathrm{~nm}$, sensing the intrinsic optical absorption contrast of hemoglobin. Microvessels in diameters down to $10 \mu \mathrm{m}$ are clearly imaged. Figure 3(b) is a snapshot of a 3D animation (Video 1) showing the mouse ear microvasculature from various perspectives. In addition to $570 \mathrm{~nm}$, laser light at $565 \mathrm{~nm}$ was also used to image the mouse ear, enabling $\mathrm{sO}_{2}$ measurement, as demonstrated previously with OR-PAM [13]. While Fig. 3(c) shows the microvasculature of a selected area, Fig. 3(d) shows the corresponding vessel-by-vessel $\mathrm{sO}_{2}$ mapping, where the arteries and veins are differentiated based on the measured $\mathrm{sO}_{2}$. On average, the $\mathrm{SO}_{2}$ values in arteries and veins were estimated to be $0.96 \pm$ 0.4 and $0.75 \pm 0.5$, respectively, consistent with previous results from OR-PAM. In the experiment, the incident laser energy on each microlens was limited to $\sim 100 \mathrm{~nJ}$ per pulse, resulting in a maximum fluence of $\sim 8 \mathrm{~mJ} / \mathrm{cm}^{2}$ per pulse on the skin surface (assuming the optical focus was $200 \mu \mathrm{m}$ below the skin), which is less than the $20-\mathrm{mJ} / \mathrm{cm}^{2}$ ANSI laser safety standard [14].

In summary, using a microlens array and a high-frequency linear ultrasound array, we developed multi-focal optical-resolution photoacoustic microscopy. The system provided volumetric imaging of optical absorption contrast in scattering biological tissue in vivo, at depths up to the optical transport mean free path ( $1 \mathrm{~mm}$ in the skin), and with optical diffraction limited lateral resolution at least as fine as $10 \mu \mathrm{m}$ (the axial resolution was $\sim 25$ $\mu \mathrm{m})$. With this high spatial resolution and multiple wavelengths, our system was capable of imaging both the concentration and oxygen saturation of hemoglobin in capillary-level microvessels in vivo. Moreover, in MFOR-PAM, the combination of multi-focal optical illumination and ultrasonic array detection enabled a significant improvement in imaging speed over existing mechanical scanning single-focal OR-PAM. In the future, by eliminating the 6:1 multiplexing in data acquisition, the imaging speed can be increased 6-fold. In addition, a more densely packed microlens array (e.g., with a spacing of $100 \mu \mathrm{m}$ ), together with optical scanning within the lens array pitch, can be used to further increase the imaging speed. To enable reflection-mode imaging, a beam combiner similar to that used in ORPAM [5] but with smaller dimensions need to be developed. In addition, a microlens array of a longer working distance, or any other multi-focal optical device with a relatively long working distance, may be needed. In clinical practice, high imaging speed is critical to reduce motion artifacts, cost, and patient discomfort. This proof-of-principle study has demonstrated that MFOR-PAM represents a promising direction for translating photoacoustic microscopy technology to the clinic.

\section{Acknowledgments}

This work was sponsored in part by NIH grants R01 EB000712, R01 EB008085, R01 CA134539, U54 CA136398, R01 EB010049, and 5P60 DK02057933. L.W. has a financial interest in Microphotoacoustics, Inc. and Endra, Inc., which, however, did not support this work. The authors appreciate Professor James Ballard's close reading of the 
manuscript. They also thank Junjie Yao, Lidai Wang, Zijian Guo, and Bin Rao for helpful discussions, Robert Berry for help on machining, and Guo Li for help in preparing the 3D figure of the system.

\section{References}

1. Stern MD. In vivo evaluation of microcirculation by coherent light scattering. Nature. 1975; 254:56-58. [PubMed: 1113878]

2. Chaigneau E, Oheim M, Audinat E, Charpak S. Two-photon imaging of capillary blood flow in olfactory bulb glomeruli. Proceedings of the National Academy of Sciences of the United States of America. 2003; 100:13081-13086. [PubMed: 14569029]

3. Laemmel E, Genet M, Le Goualher G, Perchant A, Le Gargasson JF, Vicaut E. Fibered confocal fluorescence microscopy (Cell-viZio (TM)) facilitates extended imaging in the field of microcirculation - A comparison with intravital microscopy. Journal of Vascular Research. 2004; 41:400-411. [PubMed: 15467299]

4. Wang RK, An L. Doppler optical micro-angiography for volumetric imaging of vascular perfusion in vivo. Optics Express. 2009; 17:8926-8940. [PubMed: 19466142]

5. Maslov K, Zhang HF, Hu S, Wang LV. Optical-resolution photoacoustic microscopy for in vivo imaging of single capillaries. Optics Letters. 2008; 33:929-931. [PubMed: 18451942]

6. Hu S, Wang LV. Photoacoustic imaging and characterization of the microvasculature. Journal of Biomedical Optics. 2010; 15:011101. [PubMed: 20210427]

7. Xie ZX, Jiao SL, Zhang HF, Puliafito CA. Laser-scanning optical-resolution photoacoustic microscopy. Optics Letters. 2009; 34:1771-1773. [PubMed: 19529698]

8. Rao B, Li L, Maslov K, Wang LH. Hybrid-scanning optical-resolution photoacoustic microscopy for in vivo vasculature imaging. Optics Letters. 2010; 35:1521-1523. [PubMed: 20479795]

9. Niederhauser JJ, Jaeger M, Lemor R, Weber P, Frenz M. Combined ultrasound and optoacoustic system for real-time high-contrast vascular imaging in vivo. Ieee Transactions on Medical Imaging. 2005; 24:436-440. [PubMed: 15822801]

10. Zemp RJ, Song L, Bitton R, Shung KK, Wang LV. Realtime photoacoustic microscopy in vivo with a 30-MHz ultrasound array transducer. Optics Express. 2008; 16:7915-7928. [PubMed: 18545502]

11. Song L, Maslov K, Wang LV. Section-illumination photoacoustic microscopy for dynamic 3D imaging of microcirculation in vivo. Optics Letters. 2010; 35:1482-1484. [PubMed: 20436610]

12. Song L, Maslov K, Bitton R, Shung KK, Wang LV. Fast 3-D dark-field reflection-mode photoacoustic microscopy in vivo with a $30-\mathrm{MHz}$ ultrasound linear array. Journal of Biomedical Optics. 2008; 13:054028. [PubMed: 19021408]

13. Hu S, Maslov K, Wang LHV. Noninvasive label-free imaging of microhemodynamics by opticalresolution photoacoustic microscopy. Optics Express. 2009; 17:7688-7693. [PubMed: 19399148]

14. American National Standard for Safe Use of Lasers (ANSI Z136.1-2000). The Laser Institute of America; Orlando, FL: 2000. 


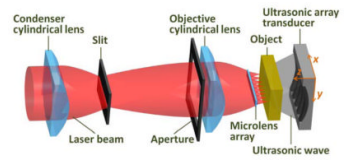

Fig. 1.

(Color online) Schematic of the multi-focal optical-resolution photoacoustic microscopy system. The widths of the slit and the aperture along $y$ are $50 \mu \mathrm{m}$ and $5 \mathrm{~mm}$, respectively. 

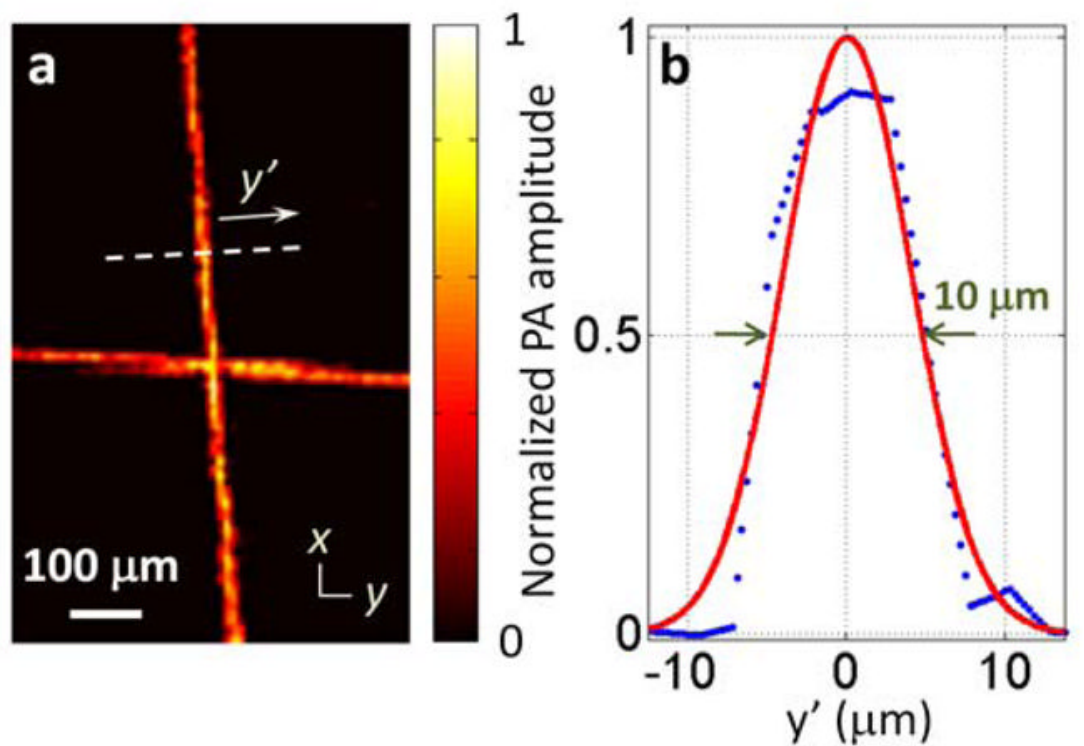

Fig. 2.

(Color online) Lateral resolution of the multi-focal optical-resolution photoacoustic microscopy system. (a) Photoacoustic (PA) maximum amplitude projection (MAP) image of two crossed 6- $\mu \mathrm{m}$ diameter carbon fibers. (b) Distribution of the PA amplitude (dots) along the dashed line in (a); the solid line is a Gaussian fitted curve. 

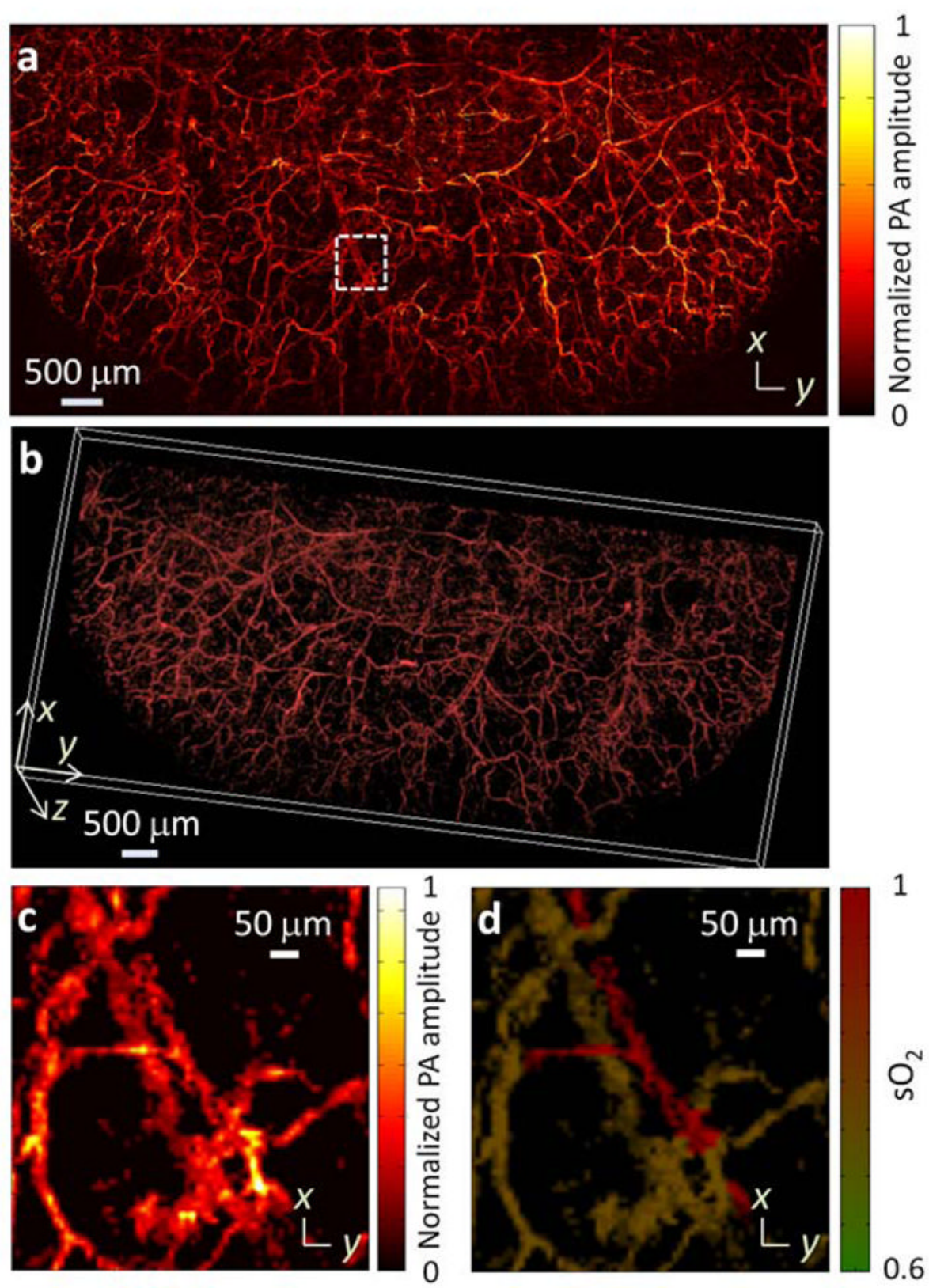

Fig. 3.

(Color online) In vivo photoacoustic image of a mouse ear microvasculature. (a) MAP image acquired at $570 \mathrm{~nm}$. (b) Snapshot of a 3D animation (Video 1) showing the microvasculature in (a). (c) Close-up of the vasculature in the dashed box in (a). (d) Oxygen saturation $\left(\mathrm{sO}_{2}\right)$ mapping for vessels in (c). 